\title{
Impact of Blood Pressure and Other Components of the Metabolic Syndrome on the Development of Cardiovascular Disease
}

\author{
Guodong Kang, MD; Lu Guo, MD*; Zhirong Guo, MD*; Xiaoshu Hu, MD**; \\ Ming Wu, MD; Zhengyuan Zhou, MPH ${ }^{\dagger}$; Hui Zhou, MD*; \\ Shijun Liu, MD*; Fengmei Chen, MD*
}

\begin{abstract}
Background: Few prospective studies have explored blood pressure (BP) and other components of the metabolic syndrome (MetS) and their interaction in the development of cardiovascular diseases (CVD) in China.

Methods and Results: A prospective study of the prevention of multiple metabolic disorders and MetS in Jiangsu province, China: 3,598 subjects were followed for a median of 6.3 years. The Asian criterion of the National Cholesterol Education Program Adult Treatment Panel III was used to define the MetS. Independent risk of the MetS and its components on developing CVD was analyzed, but only BP was associated with CVD. Incidence and risk of CVD increased with the number of MetS components. A linear association was found between the risk of CVD, BP and the number of other components (trend, $\mathrm{P}<0.01$ ). The adjusted relative risk of developing CVD was increased when BP and other components coexisted. However, the interaction of BP and other components of MetS was not significant $(P>0.05)$.
\end{abstract}

Conclusions: In Chinese, among the components of MetS BP was an independent risk factor for CVD. No significant interaction was found between BP and the other MetS components. (Circ $J$ 2010; 74: 456-461)

Key Words: Blood pressure; Cardiovascular diseases; Metabolic syndrome

$\mathbf{T}$ he metabolic syndrome (MetS), also known as insulin resistance syndrome, ${ }^{1}$ was initially described in 1988 by Reaven ${ }^{2}$ and denotes the clustering of cardiovascular risk factors, including obesity, insulin resistance, impaired glucose metabolism, dyslipidemia of high triglycerides (TG), low level of high-density lipoproteincholesterol (HDL-C) and elevated blood pressure (BP). It has become a major public health challenge worldwide. ${ }^{3}$ The best available evidence suggests that people with MetS are at increased risk of cardiovascular disease (CVD). ${ }^{4-6}$ Thus, there has been growing interest in this constellation of closely related cardiovascular risk factors.

Recently, Kahn et al raised some controversies about MetS. ${ }^{7}$ They pointed out that the definition of MetS implied that the risk of having the syndrome should be greater than the sum of its parts; that is, the factors included should have greater predictive power than other combinations. ${ }^{7}$ However, there is no obvious evidence showing that the predictive power of MetS is greater than the sum of its individual components. At the same time, whether each component of MetS shows equal risk for developing CVD, or which components of the MetS are more strongly associated with CVD, has not been sufficiently discussed. ${ }^{8,9}$ Therefore, analyzing the interaction between components of MetS and CVD could resolve this issue, but there are few similar welldesigned prospective studies in Asians, particularly in Chinese populations. ${ }^{10-13}$ The present study, in the context of the Prevention of MetS and Multi-metabolic Disorders in Jiangsu Province of China, explored these questions by analyzing the independent risk of each component for CVD and the interactions between the components of the MetS.

\section{Baseline Study}

The Prevention of MetS and Multi-metabolic Disorders in Jiangsu Province of China was an ongoing prospective study that aimed to estimate the prevalence of MetS in the province at baseline and then to evaluate the incidence of CVD and type 2 diabetes mellitus (T2DM) in the subjects in a follow-up study. Briefly, the study was conducted during early 2000 to 2004 in Jiangsu province, which is in eastern

Received June 10, 2009; revised manuscript received October 4, 2009; accepted November 3, 2009; released online January 7, 2010 Time for primary review: 54 days

Center for Diseases Control of Jiangsu Province, Nanjing, *Department of Radiology \& Public Health, Soochow University, Suzhou, **Health Bureau of Jiangsu Province, Nanjing and Center for Diseases Control of Changshu, Changshu, Jiangsu, China

Mailing address: Zhirong Guo, MD, Department of Epidemiology, Soochow University School of Radiation Medicine and Public Health,

High Education Area, Industrial Park District, Suzhou 215123, China. E-mail: guozhirong28@163.com

ISSN-1346-9843 doi:10.1253/circj.CJ-09-0422

All rights are reserved to the Japanese Circulation Society. For permissions, please e-mail: cj@j-circ.or.jp 
China and has a population of 75 million. The multi-stage sampling method was used in the baseline survey: we randomly selected 3 sites from 13 urban districts and 9 sites from 52 counties. Next, 1 community (similar to a street district or a residential committee) from each city and 1 rural township from each county were sampled randomly. Finally, individuals were randomly chosen from the selected communities and townships; only 1 participant was selected from each household, without replacement. All participants should be in the household registration at the local administrative institute. In all, 8,685 participants aged 35-74 years were randomly selected for the baseline survey from 12 primary units (each unit was $\approx 1,000-2,000$ households), stratified by age (10 years per group) and gender. There were 5,888 valid questionnaires at baseline and the overall response rate was $92.0 \%$. The investigation was supported by the local Centers for Disease Control and Prevention (CDC). Participants were asked to attend the community health station with their clinical record or health registration card after giving informed consent. The protocol was approved by the Ethics Committee of Soochow University.

Detailed information about behavioral, lifestyle and demographic factors and body measurements were obtained. Weight and height were measured for all subjects to the nearest $0.1 \mathrm{~kg}$ and $0.1 \mathrm{~cm}$, respectively. Seated systolic and diastolic BP (SBP, DBP) was measured 3 times in the right arm by mercury sphygmomanometer at 30 -s intervals after a 5-min rest; the mean value was recorded as the BP for analysis. Waist circumference (WC) of each subject was measured (at minimal respiration to the nearest $0.1 \mathrm{~cm}$ at the level of the iliac crest) twice. Blood samples were obtained after a minimum 8-h fast to measure fasting plasma glucose (FPG), TG and HDL-C levels by the glucose oxidase enzymatic method, enzymatic method and precipitation method, respectively, using an automatic biochemistry analyzer (Hitachi Inc, Tokyo Japan). All clinical and biological parameters were evaluated on the day of the physical examination. Smoking habit was defined as current smoking, ever smoking and no smoking; drinking habit was defined as current drinking, ever drinking and no drinking. In all, 5,888 participants with useful data were included at baseline. Exclusion criteria for the cohort were pregnancy, severe cancer, disability and severe psychiatric disorders.

All subjects gave informed consent at the interview.

\section{Follow-up Study}

During 2006-2008, participants who had been in the study for at least 5 years were included in the follow-up study. A total of 4,582 participants were followed and of them 4,083 were included in the second investigation with a follow-up rate of $89.11 \%$. The characteristics of the individuals who did not attend the follow-up survey, such as age, gender and metabolic variables, were similar to those included in the baseline study. Health status was checked by examination; the procedures at follow-up were similar to those used at baseline. The primary endpoints for the follow-up survey were the occurrence of CVD or T2DM. Subjects were contacted by mail or telephone. We had also established a daily monitoring system among the study team and the chronic diseases surveillance and death registration at the local CDC. For the participants who reported their own health status, we asked them for their medical records. If the subject died during the follow up, autopsy for determining the cause of death was performed at the hospital where the subject had died. In total, subjects with T2DM $(n=289)$,
CVD ( $\mathrm{n}=36)$, body mass index $<18.5 \mathrm{~kg} / \mathrm{m}^{2}(\mathrm{n}=27)$ or who were missing data $(n=133)$ at baseline were excluded from the study. Thus, as of July 2008, the 3,598 remaining subjects (males 1,451, females 2,147) were enrolled in the follow-up study. The median follow-up was 6.3 person-years (range 5-8 years). The reliability and accuracy of the collected data were checked for the study.

\section{Definition of the MetS}

The definition of the MetS used in this study was based on the Revised the National Cholesterol Education Program Adult Treatment Panel III report (NECP-R ATPIII) in Asian population. ${ }^{13}$ Specifically, the cutoff values for WC were $90 \mathrm{~cm}$ for males and $80 \mathrm{~cm}$ for females, in accordance with the modification for Asians; the threshold FPG was modified to $5.6 \mathrm{mmol} / \mathrm{L}$, which improves the predictive ability for diabetes. Elevated BP was defined as average SBP/DBP $\geq 130 / 85 \mathrm{mmHg}$ and/or current use of antihypertensive medicine. Hypertriglyceridemia was defined as serum TG $\geq 1.69 \mathrm{mmol} / \mathrm{L}$. Low HDL-C was defined as $<1.03 \mathrm{mmol} / \mathrm{L}$ in men or $<1.29 \mathrm{mmol} / \mathrm{L}$ in women. MetS was defined as the presence of 3 or more of these components.

\section{Definition of CVD}

Subjects who met 1 of the following conditions were diagnosed as having CVD. During the follow-up period, first-ever development of coronary heart disease (CHD) or stroke. The criteria for a diagnosis of CHD included interventional treatment of a coronary artery (cardiac catheterization or coronary artery bypass grafting), stable angina pectoris, unstable angina pectoris, the first occurrence of acute myocardial infarction, and congestive heart failure caused by myocardial ischemia after baseline investigation. Stroke was classified as ischemic attack or hemorrhagic attack. Peripheral vascular disease (abdominal aneurysm, operation on vessels and carotid endarterectomy) was also included as CVD. The diagnosis of CVD and the determination of its pathological type were based on standard questionnaires, signs and symptoms in the clinical history, all available clinical data and autopsy findings. Cardiovascular death during follow-up was defined as a resulting from CVD (ICD-9 codes 390-459).

\section{Statistical Analysis}

Continuous variables were tested using the t-test and noparametric test. Frequencies of categorical variables were tested using the chi-square test. A Cox proportional hazards regression model was used to evaluate the association of each component and the number of combinations of MetS components on the development of CVD. The crude relative risk (RR) and the age, gender, and smoking and drinking habit adjusted RR (aRR) were calculated. Also, the association between BP and other combinations of MetS components with CVD was determined. The trend test was carried out using the method of Breslow and Day. ${ }^{14}$

The interaction analysis between BP and other components of MetS on developing CVD was performed by comparing subjects with high BP only with the subjects with MetS ( $\geq 2$ components except for BP). In this way, different combinations of MetS included at least 3 MetS components vs non-MetS components. Therefore, subjects were divided into 2 separate factors and 4 different subgroups to determine the risks of different combinations on developing CVD and the interaction between of them:

(1) without MetS $[\mathrm{MetS}(-)]$ and without BP $[\mathrm{BP}(-)]$

(2) with MetS $[\operatorname{MetS}(+)]$ and without BP $[\mathrm{BP}(-)]$ 


\begin{tabular}{|lccc|}
\hline \multicolumn{2}{|c|}{ Table 1. Baseline Clinical and Biochemical Characteristics of the Subjects } & \\
\multicolumn{1}{c}{ Characteristic } & With MetS & Without MetS & P value \\
$\mathrm{n}$ (male) & $903(254)$ & $2,695(1,197)$ & - \\
Age (year) & $52.2 \pm 10.2$ & $49.6 \pm 9.8$ & $<0.01$ \\
WC $(\mathrm{cm})$ & $84.6 \pm 9.1$ & $74.0 \pm 7.8$ & $<0.01$ \\
SBP $(\mathrm{mmHg})$ & $138.3 \pm 20.7$ & $121.8 \pm 17.8$ & $<0.01$ \\
DBP $(\mathrm{mmHg})$ & $85.8 \pm 10.7$ & $77.9 \pm 10.2$ & $<0.01$ \\
HDL-C (mmol/L) & $1.07 \pm 0.29$ & $1.34 \pm 0.35$ & $<0.01$ \\
TG (mmol/L) & $2.10[1.11]$ & $1.18[0.62]$ & $<0.01$ \\
FPG (mmol/L) & $5.95 \pm 1.78$ & $5.14 \pm 0.90$ & $<0.01$ \\
Current smoking, $\mathrm{n}(\%)$ & $155(17.2)$ & $788(29.2)$ & $<0.01$ \\
Current drinking, $\mathrm{n}(\%)$ & $115(87.0)$ & $646(23.9)$ & $<0.01$ \\
Family history of CVD, $\mathrm{n}(\%)$ & $8(0.9)$ & $20(0.7)$ & $\mathrm{NS}$ \\
New CVD, $\mathrm{n}(\%)$ & $39(4.3)$ & $43(1.6)$ & $<0.01$ \\
\hline
\end{tabular}

Data are mean $\pm \mathrm{SD}$, percentage, or median value [interquartile range].

MetS, metabolic syndrome; WC, waist circumference; SBP, systolic blood pressure; DBP, diastolic blood pressure; HDL-C, high-density lipoprotein-cholesterol; TG, triglyeride; FPG, fasting plasma glucose; CVD, cardiovascular disease; NS, not significant.

\begin{tabular}{|c|c|c|c|c|c|c|}
\hline \multirow{2}{*}{ Risk factor } & \multicolumn{6}{|c|}{ Adjusted RR $(95 \% \mathrm{Cl})$} \\
\hline & Model 1 & $P$ value & Model 2 & $P$ value & Model 3 & $P$ value \\
\hline MetS & $2.50(1.60-3.91)$ & $<0.01$ & $2.49(1.59-3.90)$ & $<0.01$ & - & \\
\hline WC & $1.44(0.88-2.36)$ & NS & $1.44(0.88-2.37)$ & NS & $0.90(0.53-1.53)$ & NS \\
\hline BP & $2.91(1.77-4.78)$ & $<0.01$ & $2.84(1.73-4.68)$ & $<0.01$ & $2.47(1.48-4.13)$ & $<0.01$ \\
\hline Low HDL-C & $1.27(0.80-2.0)$ & NS & $1.31(0.83-2.07)$ & NS & $1.09(0.67-1.79)$ & NS \\
\hline TG & $1.84(1.19-2.84)$ & $<0.01$ & $1.84(1.19-2.85)$ & $<0.01$ & $1.28(0.78-2.08)$ & NS \\
\hline FPG & $1.58(1.0-2.50)$ & $<0.05$ & $1.55(0.98-2.45)$ & NS & $1.24(0.77-2.0)$ & NS \\
\hline
\end{tabular}

$\mathrm{RR}$, relative risk; $\mathrm{Cl}$, confidence interval; $\mathrm{BP}$, blood pressure. Other abbreviations see in Table 1.

Model 1, adjusted for age and gender; Model 2, adjusted for age, gender, smoking and drinking habits; Model 3 , adjusted for Model 2 and the other 4 components of MetS.

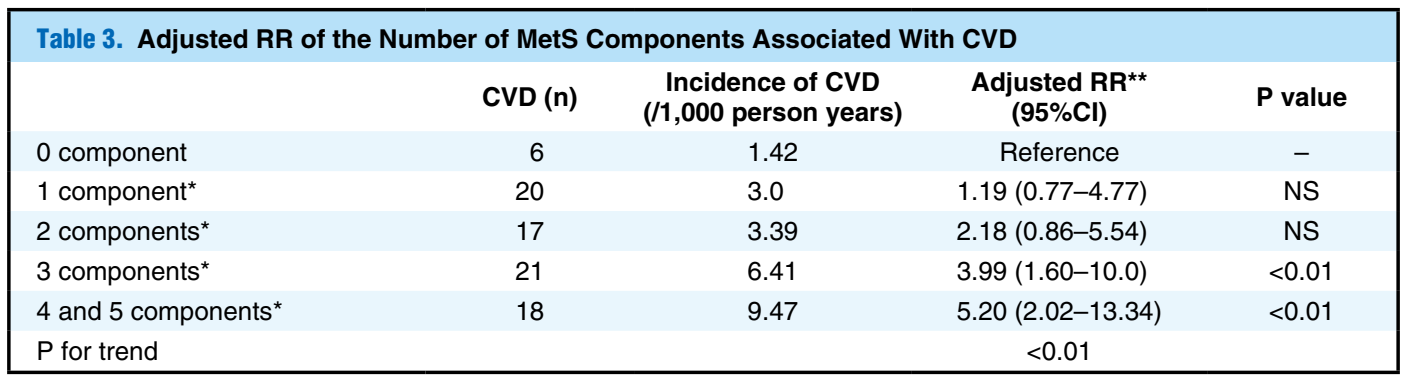

${ }^{*}$ Compared with those with 0 components of MetS; ${ }^{*}$ adjusted for age, gender, and smoking and drinking habits in a Cox proportion hazard regression model.

Abbreviations see in Tables 1,2.

(3) without MetS $[\mathrm{MetS}(-)]$ and with $\mathrm{BP}[\mathrm{BP}(+)]$

(4) with MetS $[\operatorname{MetS}(+)]$ and with $\mathrm{BP}[\mathrm{BP}(+)]$.

Interaction between 2 of the above risk factors was evaluated by 3 indexes of additive biological interaction: RERI, the relative excess risk because of the interaction; AP, the attributable proportion because of the interaction; and SI, the synergy index..$^{15}$ These measures were defined as follows:

$\mathrm{RERI}=\mathrm{RRMetS}_{(+)} \& \mathrm{BP}(+)-\mathrm{RRMetS}_{(+)}-\mathrm{RR}_{\mathrm{BP}(+)}+1$

$\mathrm{AP}=\mathrm{RERI} / \mathrm{RR}_{\mathrm{Met}} \mathrm{S}(+) \& \mathrm{BP}(+)$

$\mathrm{SI}=(\mathrm{RR} \operatorname{MetS}(+) \& \mathrm{BP}(+)-1) /\left[\left(\mathrm{RRMetS}_{(+)}-1\right)+\left(\mathrm{RRBP}_{(+)}-1\right)\right]$.

If there was no biological interaction, the confidence interval (CI) of RERI and AP included 0 , and the CI of SI contained 1.0. SPSS statistical software system for Windows version 11.5 (SPSS Inc, Chicago, IL, USA) was used to perform all statistical analyses.

\section{Results}

During the follow-up, a total of 82 subjects developed CVD. Table 1 is a comparison of the with and without MetS groups; those with MetS were older and had a high WC, SBP, DBP, TG, FPG, and low HDL-C, and also low smoking and drinking habits. There was no difference in their family histories of CVD. No significant difference int he incidence of CVD was found between males and females $(\mathrm{P}>0.05)$. Incidence of CVD in the MetS group was higher than in the 


\begin{tabular}{|c|c|c|c|c|}
\hline & CVD (n) & $\begin{array}{l}\text { Incidence of CVD } \\
(/ 1,000 \text { person years) }\end{array}$ & $\begin{array}{l}\text { Adjusted RR }{ }^{\star *} \\
(95 \% \mathrm{Cl})\end{array}$ & $P$ value \\
\hline 0 component & 6 & 1.42 & Reference & \\
\hline BP only* & 12 & 5.82 & $2.97(1.10-8.04)$ & $<0.01$ \\
\hline $\mathrm{BP}+1$ other component ${ }^{\star}$ & 11 & 4.64 & $2.56(0.94-6.96)$ & NS \\
\hline $\mathrm{BP}+2$ other components* & 18 & 8.08 & $4.65(1.82-11.85)$ & $<0.01$ \\
\hline $\mathrm{BP}+3$ or 4 other components* & 18 & 10.56 & $5.58(2.17-14.38)$ & $<0.01$ \\
\hline$P$ for trend & & & $<0.01$ & \\
\hline $1-4$ components without $\mathrm{BP}^{*}$ & 17 & 2.00 & $1.47(0.56-3.83)$ & NS \\
\hline 3 or 4 components without $\mathrm{BP}^{*}$ & 3 & 2.41 & $1.74(0.39-7.68)$ & NS \\
\hline
\end{tabular}

${ }^{*}$ Compared with 0 components of MetS; ${ }^{* *}$ adjusted for age, gender, and smoking and drinking habits in a Cox proportion hazard regression model.

Abbreviations see in Tables 1,2.

Table 5. Interaction Analysis Between BP and 2, 3 or 4 Other Components of the MetS Associated With CVD

\begin{tabular}{|c|c|c|c|c|}
\hline Interpretation & $\operatorname{CVD}(n)$ & $\begin{array}{l}\text { Incidence of CVD } \\
(/ 1,000 \text { person years) }\end{array}$ & Adjusted $\mathbf{R R}^{\star *}$ & $P$ value \\
\hline MetS (-) and BP (-) & 14 & 1.59 & Reference & - \\
\hline MetS $(+)$ and BP $(-)^{*}$ & 9 & 2.31 & $1.49(0.64-3.47)$ & NS \\
\hline $\operatorname{MetS}(-)$ and BP $(+)^{*}$ & 12 & 5.82 & $2.37(1.07-5.23)$ & $<0.05$ \\
\hline MetS $(+)$ and BP $(+)^{*}$ & 36 & 9.16 & $4.34(2.30-8.18)$ & $<0.01$ \\
\hline
\end{tabular}

${ }^{*}$ Compared with 1 component of MetS other than BP; **adjusted for age, gender, and smoking and drinking habits in a Cox proportion hazard regression model.

MS (-), 1 component of MetS other than BP; MS (+), 2, 3 or 4 components of MetS except BP; BP (-), without BP;

$\mathrm{BP}(+)$, with BP only. Other abbreviations see in Tables 1,2 .

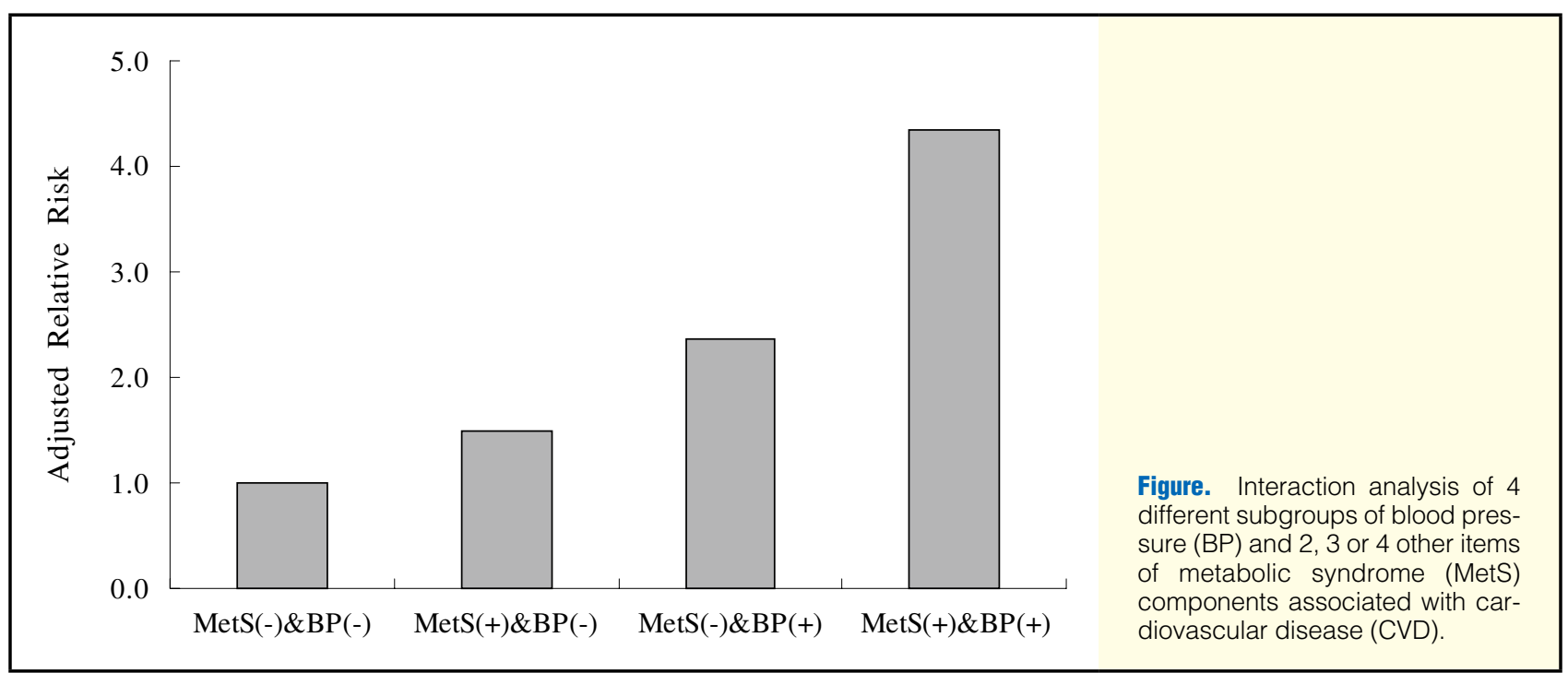

non-MetS group.

Table 2 shows the aRR of each component of the MetS for predicting CVD in the Cox hazard proportion regression model. After adjusting for baseline age and gender, excepting WC and low HDL-C, the aRR of MetS was 2.50 (95\% CI 1.60-3.91), and BP (aRR 2.91; 95\%CI 1.77-4.78), TG (aRR 1.84; 95\%CI 1.19-2.84) and FPG (aRR 1.58; 95\%CI 1.00 2.50) were associated with CVD (Model 1). Next, smoking and drinking habits were further adjusted, and we found that MetS (aRR 2.49; 95\%CI 1.59-3.90), BP (aRR2.84; 95\%CI 1.73-4.68) and TG (aRR1.84; 95\%CI 1.19-2.85) were significantly associated with CVD (Model 2). After mutually adjusting for each of the MetS components, the result indi- cated that only BP was still independently associated with CVD (aRR of BP 2.47; 95\%CI 1.48-4.13); the other components of MetS had no relationship with CVD (Model 3). Meanwhile, subjects with 1-4 or 5 components of the MetS had an increased aRR for developing CVD from 1.34 (95\% CI $0.84-2.14)$ to $5.20(95 \% \mathrm{CI} 2.02-13.34)$ (trend $\mathrm{P}<0.01$ ) compared with those without any components (Table 3 ).

Accordingly, we further analyzed the risk for CVD of BP combined with other MetS components. As shown in Table 4, in contrast with those subjects without any MetS components, the incidence of CVD increased from 1.42 per 1,000 person years to 10.56 per 1,000 person years. Also, a linear association was found between BP and the number of 
other components for the aRR (from 2.97 to 5.58) of CVD (trend, $\mathrm{P}<0.01$ ). In particular, baseline subjects with MetS without BP (aRR 1.74; 95\%CI 0.39-7.68) were no longer at significant risk for CVD after adjustment for age, gender, and smoking and drinking habits.

To explore the interaction between BP and the other MetS components with CVD, 2 factors were dichotomized according to the definition of NECP-R ATPIII. Subjects who had 2 or more MetS components other than high BP, including high WC, TG, FPG and low HDL-C, were considered as factor A against subjects who had high BP only, who were considered as factor B. Next, the 2 separate factors, which comprised 4 different subgroups, were analyzed in pairs in the Cox proportion hazard regression model. As shown in Table 5 and Figure, after adjusting for age, gender, and smoking and drinking habits, the aRR between CVD and BP only was 2.37 (95\%CI 1.07-5.23), and the aRR between CVD and a combination of 2, 3 or 4 other components of the MetS was 1.49 (95\%CI 0.64-3.47). When the 2 factors coexisted the aRR was 4.34 (95\%CI 2.30-8.18). Also, the index of biological interaction, RERI, was 1.49 (95\% CI -0.783.76), AP was $0.34(95 \% \mathrm{CI}-0.13-0.81)$ and SI was 1.80 (95\%CI 0.59-5.51), which indicated no observable biological interaction between the 2 risk factors.

\section{Discussion}

MetS has been proved to be a risk factor for CVD in a series of studies. ${ }^{16-18}$ In the present study, we found that the MetS increased the incidence of CVD after 5-8 years of follow-up; subjects with the MetS had a 2.45-fold increased of CVD compared with those without MetS. Lower or higher RR is reported in different Asian and Western studies, which may relate to differences in the study populations, the prevalence of the individual components of the MetS, use of different definitions and varied follow-up. ${ }^{16-18}$ Our study still confirmed that determining the presence of the MetS is a simple and useful tool for predicting CVD, although it loses some information when its components are dichotomized. ${ }^{19,20}$

Increasing research concerning the independent association of the MetS and its components for predicting CVD has been reported. ${ }^{21}$ In Swedish men, after adjusting for traditional risk factor and 5 MetS components, the relationship between the MetS and CVD disappeared. ${ }^{22}$ In the British Women Heart and Health Study, the MetS was not a predictor of CVD after adjusting for its components, and only BP and low HDL-C had a relationship with CVD. ${ }^{23}$ In a 13 -year follow-up prospective cohort study in Finns, only low HDL$\mathrm{C}$ was independently associated with CVD. ${ }^{24}$ Similarly, our study also found that the relationship between the MetS and CVD was not significant after adjusting for general risk and for MetS components, which suggests the MetS cannot provide more predictive information of CVD independent of its single components.

Which component of the MetS was associated with CVD to a larger degree? In the Asia-Pacific region, up to $66 \%$ of some subtypes of CVD can be attributed to hypertension..$^{25}$ For subjects in the National Health and Nutrition Examination Survey III, when adjusted for general risk factors and MetS components, the prediction of the MetS was not significant; nevertheless, BP and low HDL-C were associated with chronic heart disease. ${ }^{26}$ In the $4 \mathrm{~S}$ Group and the AFCAPS/ TexCAPS Research Group study, using subjects who received placebo treatment, it was found that only BP had a significant relationship with CHD after adjusting for the other compo- nents of the MetS. ${ }^{16}$ Tanomsupet et al documented that when the MetS components are adjusted mutually, components of the MetS other than BP are not related to CVD in Thais. ${ }^{18}$ In a study in Taiwan, hypertension was the most important component of the MetS in the association with ischemic heart disease in subjects with T2DM. ${ }^{27}$ In our study, after adjusting for general risks, the aRRs of the MetS and BP in predicting CVD were closely associated (Model 2 in Table 2). When the MetS components were further adjusted for each other (Model 3 in Table 2), we found that none but BP was an independent CVD risk factor. Therefore, both previous studies and our study indicate that BP, especially in mid-life, is the main risk factor for developing CVD. ${ }^{28,29}$

It is already clear that MetS components, such as central obesity, FPG, dyslipidemia and BP, are important risk factors for CVD. Theoretically, when a number of risk factors occur together in an individual, the risk of an additive effect should be more than the sum of each risk factor. That is to say, the MetS should theoretically demonstrate a higher predictive value than BP and the other components on developing CVD. In the Hisayama Study, hypertensive subjects with the MetS exceeded the risk of CVD more than either hypertensive subjects without the MetS or normotensive subjects with the MetS. ${ }^{30}$ In French middle-aged subjects without a history of CVD, no significant interaction was found to increase the risk of all-cause mortality among hypertensive and normotensive subjects. ${ }^{31}$ In our study, only BP had an independent association with CVD. In contrast, individuals with a combination of 2,3 or 4 factors other than $\mathrm{BP}$ were not associated with CVD risk. Although BP and other components coexisted, the aRR for CVD exceeded the 2 separate factors alone, and the interaction of the 2 separate factors was not significant. That result shows that BP and the other components do not have an additive effect in predicting CVD. It may be because the MetS components have a common physiologic foundation, such as insulin resistance, ${ }^{32}$ inflammation ${ }^{33}$ or central obesity, ${ }^{34}$ or that they are closely related to each other, but BP was the predominant risk factor of the MetS on development of CVD. So far, there is no distinguished evidence suggesting an interaction between $\mathrm{BP}$ and other MetS components in relation to CVD.

\section{Study Limitations}

The proportions of men and women were not ideal, as there were more women than men at baseline. Therefore, the association between the MetS and its components and CVD in both sexes should be further confirmed in later research. On the other hand, different combinations of the MetS may lead to different classifications of T2DM or CVD, ${ }^{35}$ which needs to be investigated in a prospective cohort study with longterm follow-up and large study sample.

In conclusion, we have shown that after a median 6.3 person years of follow-up, the MetS has a predictive value for CVD independent of traditional risks. However, the risk of each MetS component in predicting CVD differs. BP was independently associated with CVD. When BP and other MetS components coexisted, the MetS patient should have an increased risk of CVD, but we could not find an obvious interaction between $\mathrm{BP}$ and other MetS components.

\section{Acknowledgments}

Funding was provided by the Scientific Research Fund of Ministry of Public Health, China. This study was supported in part by the CDC of Jiangsu province, Nanjing, China and the local community health station. 


\section{References}

1. DeFronzo RA. Insulin resistance: A multifaceted syndrome responsible for NIDDM, obesity, hypertension, dyslipidaemia and atherosclerosis. Neth J Med 1997; 50: 191-197.

2. Reaven GM. Banting Lecture 1988: Role of insulin resistance in human disease. Diabetes 1988; 37: 1595-1607.

3. Eckel RH, Grundy SM, Zimmet PZ. The metabolic syndrome. Lancet 2005; 365: 1415-1428.

4. Grundy SM, Brewer HB Jr, Cleeman JI, Smith SC Jr, Lenfant C; American Heart Association; National Heart Lung, and Blood Institute. Definition of metabolic syndrome: Report of the National Heart, Lung, and Blood Institute/American Heart Association conference on scientific issues related to definition. Circulation 2004; 109: $433-438$.

5. Lakka HM, Laaksonen DE, Lakka TA, Niskanen LK, Kumpusalo E, Tuomilehto $\mathrm{J}$, et al. The metabolic syndrome and total and cardiovascular disease mortality in middle-aged men. JAMA 2002; 288: $2709-2716$

6. Hoshino A, Nakamura T, Enomoto S, Kawahito H, Kurata H, Nakahara Y, et al. Prevalence of coronary artery disease in Japanese patients with cerebral infarction: Impact of metabolic syndrome and intracranial large artery atherosclerosis. Circ J 2008; 72: 404 408.

7. Kahn R, Buse J, Ferrannini E, Stern M. The metabolic syndrome: Time for a critical appraisal: Joint statement from the American Diabetes Association and the European Association for the Study of Diabetes. Diabetes Care 2005; 28: 2289-2304.

8. Tikhonoff V, Casiglia E, Tikhonoff V, Casiglia E. Metabolic syndrome: Nothing more than a constellation? Eur Heart J 2007; 28: $780-781$.

9. Greenland P, Greenland P. Critical questions about the metabolic syndrome. Circulation 2005; 112: 3675-3676.

10. Sone H, Mizuno S, Fujii H, Yoshimura Y, Yamasaki Y, Ishibashi $\mathrm{S}$, et al. Is the diagnosis of metabolic syndrome useful for predicting cardiovascular disease in Asian diabetic patients? Analysis from the Japan Diabetes Complications Study. Diabetes Care 2005; 28: $1463-1471$.

11. Chen HJ, Bai CH, Yeh WT, Chiu HC, Pan WH. Influence of metabolic syndrome and general obesity on the risk of ischemic stroke. Stroke 2006; 37: 1060-1064.

12. Chien KL, Hsu HC, Sung FC, Su TC, Chen MF, Lee YT, et al. Metabolic syndrome as a risk factor for coronary heart disease and stroke: An 11-year prospective cohort in Taiwan community. Atherosclerosis 2007; 194: 214-221.

13. Expert Panel on Detection EaToHBCiA. Executive Summary of The Third Report of The National Cholesterol Education Program (NCEP) Expert Panel on Detection, Evaluation, And Treatment of High Blood Cholesterol In Adults (Adult Treatment Panel III). JAMA 2001; 285: 2486-2497.

14. Breslow NE, Day NE. Statistical methods in cancer research. Volume I: The analysis of case-control studies. IARC Scientific Publications, 1980; 5-338.

15. Hallqvist J, Ahlbom A, Diderichsen F, Reuterwall C. How to evaluate interaction between causes: A review of practices in cardiovascular epidemiology. J Intern Med 1996; 239: 377-382.

16. Girman CJ, Rhodes T, Mercuri M, Pyorala K, Kjekshus J, Pedersen $\mathrm{TR}$, et al. The metabolic syndrome and risk of major coronary events in the Scandinavian Simvastatin Survival Study (4S) and the Air Force/Texas Coronary Atherosclerosis Prevention Study (AFCAPS/TexCAPS). Am J Cardiol 2004; 93: 136-141.

17. Hu G, Qiao Q, Tuomilehto J, Balkau B, Borch-Johnsen K, Pyorala $\mathrm{K}$. Prevalence of the metabolic syndrome and its relation to allcause and cardiovascular mortality in nondiabetic European men and women. Arch Intern Med 2004; 164: 1066-1076.

18. Tanomsup S, Aekplakorn W, Sritara P, Woodward M, Yamwong $\mathrm{S}$, Tunlayadechanont $\mathrm{S}$, et al. A comparison of components of two definitions of the metabolic syndrome related to cardiovascular disease and all-cause mortality in a cohort study in Thailand. Diabetes Care 2007; 30: 2138-2140.
19. Stern MP, Williams K, Gonzalez-Villalpando C, Hunt KJ, Haffner $\mathrm{SM}$. Does the metabolic syndrome improve identification of individuals at risk of type 2 diabetes and/or cardiovascular disease? Diabetes Care 2004; 27: 2676-2681 [erratum Diabetes Care 2005; 28: 238].

20. Wannamethee SG, Shaper AG, Lennon L, Morris RW. Metabolic syndrome vs Framingham Risk Score for prediction of coronary heart disease, stroke, and type 2 diabetes mellitus. Arch Intern Med 2005; 165: 2644-2650.

21. Oda E. The metabolic syndrome (emperor) wears no clothes: Response to Kahn. Diabetes Care 2006; 29: 2566.

22. Sundstrom J, Riserus U, Byberg L, Zethelius B, Lithell H, Lind L, et al. Clinical value of the metabolic syndrome for long term prediction of total and cardiovascular mortality: Prospective, population based cohort study. BMJ 2006; 332: 878-882.

23. Lawlor DA, Smith GD, Ebrahim S. Does the new International Diabetes Federation definition of the metabolic syndrome predict CHD any more strongly than older definitions? Findings from the British Women's Heart and Health Study. Diabetologia 2006; 49: $41-48$.

24. Wang J, Ruotsalainen S, Moilanen L, Lepisto P, Laakso M, Kuusisto $\mathrm{J}$, et al. The metabolic syndrome predicts cardiovascular mortality: A 13-year follow-up study in elderly non-diabetic Finns. Eur Heart $J$ 2007; 28: 857-864.

25. Martiniuk AL, Lee CM, Lawes CM, Ueshima H, Suh I, Lam TH, et al. Hypertension: Its prevalence and population-attributable fraction for mortality from cardiovascular disease in the Asia-Pacific region. J Hypertens 2007; 25: 73-79.

26. Alexander CM, Landsman PB, Teutsch SM, Haffner SM; Third National Health and Nutrition Examination Survey (NHANES III); National Cholesterol Education Program (NCEP). NCEP-defined metabolic syndrome, diabetes, and prevalence of coronary heart disease among NHANES III participants age 50 years and older. Diabetes 2003; 52: 1210-1214

27. Tseng CH, Chong CK, Tseng CP, Shau WY, Tai TY. Hypertension is the most important component of metabolic syndrome in the association with ischemic heart disease in Taiwanese type 2 diabetic patients. Circ J 2008; 72: 1419-1424.

28. Launer LJ, Masaki K, Petrovitch H, Foley D, Havlik RJ. The association between midlife blood pressure levels and late-life cognitive function: The Honolulu-Asia Aging Study. JAMA 1995; 274: $1846-1851$

29. Qiu C, Winblad B, Fratiglioni L. The age-dependent relation of blood pressure to cognitive function and dementia. Lancet Neurol 2005; 4: 487-499.

30. Ninomiya T, Kubo M, Doi Y, Yonemoto K, Tanizaki Y, Rahman M, et al. Impact of metabolic syndrome on the development of cardiovascular disease in a general Japanese population: The Hisayama study. Stroke 2007; 38: 2063-2069.

31. Pannier B, Thomas F, Bean K, Jego B, Benetos A, Guize L, et al. The metabolic syndrome: Similar deleterious impact on all-cause mortality in hypertensive and normotensive subjects. J Hypertens 2008; 26: $1223-1228$

32. Alberti KG, Zimmet PZ. Definition, diagnosis and classification of diabetes mellitus and its complications. Part 1: Diagnosis and classification of diabetes mellitus provisional report of a WHO consultation. Diabet Med 1998; 15: 539-553.

33. Ishikawa S, Kayaba K, Gotoh T, Nakamura Y, Kajii E, Ishikawa S, et al. Metabolic syndrome and C-reactive protein in the general population: JMS Cohort Study. Circ J 2007; 71: 26-31.

34. Alberti KG, Zimmet P, Shaw J, Alberti KGMM, Zimmet P, Shaw J. Metabolic syndrome--a new world-wide definition: A Consensus Statement from the International Diabetes Federation. Diabet Med 2006; 23: 469-480.

35. Liu SJ, Guo ZR, Hu XS, Wu M, Chen FM, Kang GD, et al. Risks for type-2 diabetes associated with the metabolic syndrome and the interaction between impaired fasting glucose and other components of metabolic syndrome the study from Jiangsu, China of 5 years follow-up. Diabetes Res Clin Pract 2008; 81: 117-123. 